\title{
ÉPOCAS DE COLHEITA E TRATAMENTOS PRÉ-GERMINATIVOS PARA SUPERAÇÃO DA DORMÊNCIA DE SEMENTES DE Mimosa caesalpiniifolia BENTH. ${ }^{1}$
}

Josivan Viana Leal ${ }^{2}$, Edna Ursulino Alves ${ }^{3}$, Riselane de Lucena Alcântara Bruno ${ }^{3}$, Walter Esfrain Pereira ${ }^{3}$, Anarlete Ursulino Alves ${ }^{4}$, Evio Alves Galindo ${ }^{2}$ e Adriana Ursulino Alves ${ }^{4}$

\begin{abstract}
RESUMO - Este trabalho teve por objetivo avaliar a influência da época de colheita e de tratamentos prégerminativos na germinação e vigor de sementes de Mimosa caesalpiniifolia Benth. (sabiá). Para tanto, realizouse um experimento no Laboratório de Análise de Sementes do CCA-UFPB, em Areia, PB, em delineamento inteiramente ao acaso, com os tratamentos distribuídos em esquema fatorial 2 × 2 x 14, com os fatores ano de colheita (2002 e 2003), sementes dentro ou fora do craspédio e tratamentos pré-germinativos, com quatro repetições de 50 sementes. Os tratamentos pré-germinativos consistiram na imersão em água, à temperatura ambiente, por $12,24,36,48$ e $60 \mathrm{~h}$; imersão em água quente, à temperatura de $70,80,90$ e $100^{\circ} \mathrm{C}$, por $1 \mathrm{~min}$; imersão em água quente, à temperatura de $70,80,90$ e $100^{\circ} \mathrm{C}$, até o resfriamento total; e testemunha (sementes intactas). As características analisadas foram: porcentagem, primeira contagem e índice de velocidade de emergência das plântulas. Os tratamentos de imersão em água quente $\left(80^{\circ} \mathrm{C}\right)$ até o resfriamento total, nas sementes de 2002 , seguido do mesmo tratamento a $90^{\circ} \mathrm{C}$, em sementes que foram semeadas fora do craspédio, mostraram-se mais adequados para condução dos testes de germinação e vigor em sementes de M. caesalpiniifolia. As sementes semeadas fora do craspédio foram as mais apropriadas para avaliação da qualidade fisiológica, apresentando também maior homogeneidade do processo germinativo, independentemente do ano avaliado.
\end{abstract}

Palavras-chave: Mimosa caesalpiniifolia, sabiá e craspédio.

\section{HARVEST TIME AND PRE-GERMINATING TREATMENTS TO OVERCOME DORMANCY ON GERMINATION AND VIGOR OF Mimosa caesalpiniifolia BENTH. SEEDS}

\begin{abstract}
The purpose of this research was to evaluate the influence of harvest time and pre-germinating treatments on germination and vigor of seeds of Mimosa caesalpiniifolia Benth. Thus, an experiment was carried out in the Laboratory of Seed Analysis of CCA-UFPB, in Areia-PB, using a completely randomized design in a $2 \times 2 \times 14$ factorial scheme, with the factors being year of harvest (2002 and 2003), seeds inside or outside the craspedium and pre-germinating treatments, with four repetitions of 50 seeds per treatment. The treatments consisted of immersion in water at ambient temperature for 12, 24, 36, 48 and 60 hours; immersion in hot water at 70,80, 90 and $100^{\circ} \mathrm{C}$ during one minute; immersion in hot water at 70, 80, 90 and $100^{\circ} \mathrm{C}$ until total cooling and check (intact seeds). The analyzed characteristics were: percentage, first counting and seedling emergence speed index. Immersion in hot water $\left(80^{\circ} \mathrm{C}\right)$ up to total cooling, using the 2002 seeds followed by the same treatment at $90^{\circ} \mathrm{C}$, with seeds sown outside the craspedium were more adequate for the germination and vigor tests in M. caesalpiniifolia. The seeds sown outside the craspedium were more appropriate for evaluation of seed physiological quality, regardless of the evaluated year.
\end{abstract}

Keywords: Mimosa caesalpiniifolia, craspedium and germination.

\footnotetext{
${ }^{1}$ Recebido em 07.11.2006 e aceito para publicação em 20.02.2008.

${ }^{2}$ Graduação em Agronomia.CCA-UFPB, Areia-PB, Areia-PB. E-mail : <josivan@ onwave.com.br > e <eviogalindoea@ hotmail.com>..

${ }^{3}$ Departamento de Fitotecnia. CCA-UFPB, Areia-PB. Cx. Postal 2, 58397-000 Areia-PB. E-mail : <ednaursulino@ cca.ufpb.br>, $<$ lane@cca.ufpb.br>e<wep@pq.cnpq.br>.

${ }^{4}$ Programa de Pós-Graduação em Agronomia da UNESP-Jaboticabal-SP. E-mail : <urlino@ hotmail.com>e < adusp@ hotmail.com>.
} 


\section{INTRODUÇÃO}

Mimosa caesalpiniifolia Benth. é uma árvore de pequeno porte pertencente à família Mimosaceae, nativa da Região Nordeste do Brasil, que vem sendo progressivamente cultivada e, hoje, difundida do Maranhão ao Rio de Janeiro (RIZZINI, 1971; RIBEIRO, 1984). A planta possui desenvolvimento rápido, e sua madeira, por ser pesada, dura, compacta e durável, é empregada na confecção de estacas, mourões e dormentes. A folhagem pode ser utilizada como forragem seca ou fenada, especialmente na época da seca (RIZZINI, 1971).

No que concerne à floração, fica evidente a sua precocidade, pois chega a florescer antes de completar 1 ano de idade (ANDRADE, 1956 citado por COSTA, 1983). O fruto é um legume articulado que, ao atingir a maturidade, dispõe de um mecanismo de dispersão que facilita a separação do fruto em pequenos segmentos quadrangulares, unisseminados, denominados craspédios.

As sementes apresentam problemas de dormência devido à impermeabilidade do tegumento à água, que é a causa mais comum nas sementes de espécies de várias famílias, como Leguminosae, Malvaceae, Geraniaceae, Chenopodiaceae, Convolvulaceae, Solanaceae e Liliaceae, entre outras (KRAMER e KOZLOWSKI, 1972; POPINIGIS, 1985; CÍCERO, 1986). Carvalho e Nakagawa (2000) enfatizaram que esse tipo de dormência é encontrado com maior freqüência em Leguminosae.

A dormência é uma característica que favorece a sobrevivência da planta no ambiente, mas é um problema quando se deseja a propagação dessas espécies para fins de cultivo e produção de mudas, entre outros. Isso ocorre devido ao fato de a germinação das sementes tornar-se lenta e desuniforme; nesse caso, é necessário que se estabeleça, primariamente à semeadura, tratamentos que possam aumentar e uniformizar a germinação (LEMOS FILHO et al., 1997).

Sob condições naturais, a escarificação pode-se dar pelo aquecimento úmido ou seco do solo, em temperaturas alternadas, o que permitiria a entrada de água para o interior da semente. Esse processo pode ocorrer, também, pela ação de ácidos, quando as sementes são ingeridas por animais dispersores, além da ação dos microrganismos do solo (VAZQUEZ-YANES e OROZCO-SEGOVIA, 1993).

R. Árvore, Viçosa-MG, v.32, n.2, p.203-210, 2008
A exposição de sementes com dormência tegumentar a temperaturas elevadas é um meio eficiente para a sua germinação (HARTMANN e KESTER, 1978). Aágua quente ou fervente é bastante utilizada e tem-se mostrado efetiva na superação de dormência das sementes de várias espécies florestais de leguminosas, como Acacia spp. (WILLAN, 1990; BRASIL, 1992), Acacia senegal (L.) Willd e Parkinsonia aculeata L. (TORRES e SANTOS, 1994), Acacia mangium Willd (LIMA e GARCIA, 1996), Leucaena leucocephala (Lam.) de Wit (TELES et al., 2000) e Peltophorum dubium (Sprengel) Taubert (OLIVEIRA et al., 2003).

A eficiência dos tratamentos com água quente foi comprovada por Ribas et al. (1996), quando obtiveram germinação de sementes de Mimosa bimucronata (DC.) O. Kuntze entre 97 e $99 \%$ ao serem submetidas a tratamentos de imersão em água quente $\left(80^{\circ} \mathrm{C}\right)$ por 1 e 5 min e imersão em água à temperatura de $80^{\circ} \mathrm{C}$, seguido de esfriamento natural das sementes por 24 h. Também, Scheffer-Basso e Vendrusculo (1997) indicaram a imersão em água quente, durante 5 min, como um método eficiente, fácil e barato de superação da dureza das sementes de Adesmia araujoi Burk. (Fabaceae). Barbosa et al. (2004) constataram 89\% de germinação após a imersão de sementes de Ochroma lagopus Sw. (Bombacaceae) em água a $80^{\circ} \mathrm{C}$ até o resfriamento total.

Martins-Corder e Borges Junior (1999) recomendaram a autoclavagem por períodos entre 20 e 25 min como um método eficiente para superação de dormência e desinfestação de sementes de Acacia mearnsii De Wild. No entanto, enfatizaram que períodos de autoclavagem superiores a $25 \mathrm{~min}$ ocasionaram a morte do embrião das referidas sementes.

Apesar de ser um método vantajoso, de baixo custo e eficiente para superar a dormência de sementes de leguminosas, a água fervente tem apresentado resultados inferiores (RODRIGUES et al., 1990). Em sementes de Senna macranthera (Colladon) Irwin \& Barneby, os tratamentos com água quente foram menos eficazes do que aqueles com ácido sulfúrico (SANTARÉM e ÁQUILA, 1995). De forma semelhante, nas sementes de Achras sapota L. a imersão em água a $60{ }^{\circ} \mathrm{C}$ por 1,2 e 3 min não foi recomendada como tratamento prégerminativo (AZERÊDO et al., 2002). Também, foi constatada a inibição da germinação em sementes de Enterolobium contortisiliquum (Vell.) Morong 
(CANDIDO et al., 1982), Stryphnodendron pulcherrimum (Willd.) Hochr (VARELA et al., 1991), Mimosa caesalpiniaefolia Benth (MARTINS et al., 1992) e de Copaifera langsdorfii Desf. (PEREZ e PRADO, 1993).

De acordo com Eira et al. (1993), vantagens e desvantagens foram detectadas em todos esses tratamentos, de modo que cada um deve continuar sendo estudado, considerando-se também o custo efetivo e sua facilidade de execução. Além disso, em um mesmo lote pode haver sementes com diferentes níveis de dormência. Assim, o método empregado deve ser efetivo na superação da dormência das sementes sem prejudicar aquelas com baixos níveis de dormência.

Diante do exposto, o objetivo deste trabalho foi avaliar o efeito de diferentes tratamentos prégerminativos sobre a germinação e vigor de sementes de M. caesalpinifolia.

\section{MATERIAL E MÉTODOS}

O efeito de diferentes tratamentos pré-germinativos sobre a germinação e o vigor de sementes de $M$. caesalpiniifolia foi conduzido entre outubro/2003 e maio/2004, no Laboratório de Análise de Sementes do Departamento de Fitotecnia do Centro de Ciências Agrárias (CCA-UFPB), Campus de Areia, da Universidade Federal da Paraíba. As sementes foram obtidas no laboratório, provenientes de 10 árvores com características de matrizes, no Município de Areia, PB, as quais foram coletadas em 2002 e acondicionadas em câmara fria, sob temperatura em torno de $10^{\circ} \mathrm{C}$ e umidade relativa do ar de $60 \%$; também, foram utilizadas sementes recém-colhidas do ano de 2003, ambas coletadas a partir de frutos completamente maduros.

Após a colheita, os frutos foram postos para secar ao sol e, em seguida, submetidos ao método manual de debulha, que consistiu na aplicação de golpes com uma vara de $50 \mathrm{~cm}$ de comprimento e $14 \mathrm{~cm}$ de diâmetro, sobre um amontoado de vagens secas ao sol durante $5 \mathrm{~min}$. Durante esse processo, os frutos permaneceram em sacos de pano grosso e resistente, de modo a ficarem comprimidos e evitando a dispersão das sementes. O peso de frutos por tratamento foi de aproximadamente $150 \mathrm{~g}$.

As sementes, dentro ou fora dos craspédios, foram submetidas aos seguintes tratamentos pré-germinativos: imersão em água à temperatura ambiente por 12, 24, 36,48 e $60 \mathrm{~h}$; imersão em água quente nas temperaturas de $70,80,90 \mathrm{e} 100^{\circ} \mathrm{C}$ por 1 min e posteriormente resfriadas em água fria (choque térmico); imersão em água quente nas temperaturas de $70,80,90$ e $100^{\circ} \mathrm{C}$ até o resfriamento total e o testemunha (sementes intactas).

O teste de emergência foi instalado em casa de vegetação do CCA-UFPB, em outubro de 2003, tendo como substrato areia previamente esterilizada em autoclave, utilizando-se como recipientes bandejas plásticas com dimensões de $45 \mathrm{~cm}$ de comprimento x $30 \mathrm{~cm}$ de largura x $6 \mathrm{~cm}$ de altura. Posteriormente, as sementes dentro e fora dos craspédios foram semeadas a uma profundidade de aproximadamente $1,5 \mathrm{~cm}$, em posição horizontal.

O delineamento estatístico utilizado foi o inteiramente casualizado, considerando-se para o teste de primeira contagem de emergência quatro repetições de 50 sementes para cada tratamento. Nos testes de emergência e vigor (IVE), utilizou-se o mesmo delineamento em esquema fatorial $2 \times 2 \times 14$, os quais consistiram de sementes dentro e fora dos craspédios, submetidas à imersão em água fria e água quente, sendo, em água fria, por cinco períodos de tempo diferentes e quatro períodos para os tratamentos com água quente, além da testemunha (sementes intactas contidas nos craspédios).

As contagens foram feitas diariamente, a partir de plântulas completamente emersas, ou seja, cotilédones acima do substrato, tendo início no terceiro dia e estendendo-se até o $22^{\circ}$ dia após a instalação. A germinação, para as sementes dentro ou fora dos craspédios, estabilizou-se no $16^{\circ}$ dia do início do teste, razão pela qual se levou em conta esta data para indicativo de contagem final das plântulas emersas. Para avaliação do efeito dos tratamentos, adotaram-se as variáveis descritas a seguir.

Porcentagem de germinação: consideraramse, ao final do teste (16 dias), as plântulas normais, ou seja, aquelas que apresentavam todas as estruturas essenciais perfeitas (BRASIL, 1992).

Primeira contagem de emergência: corresponde à porcentagem acumulada de plântulas emersas até o oitavo dia após o início do teste.

Índice de velocidade de emergência (IVE): determinado mediante a contagem diária do número de plântulas emersas a partir do $3^{\circ}$ até $16^{\circ}$ dia após a semeadura, sendo o índice calculado pela fórmula proposta por Maguire (1962).

R. Árvore, Viçosa-MG, v.32, n.2, p.203-210, 2008 


$$
I V E=\frac{E_{1}}{N_{1}}+\frac{E_{2}}{N_{2}}+\ldots+\frac{E_{n}}{N_{n}}
$$

em que: IVE = índice de velocidade de emergência; $\mathbf{E}_{1}, \mathbf{E}_{2}, \mathbf{E}_{\mathrm{n}}=$ número de plântulas normais, computadas na primeira, na segunda e na última contagem; e $\mathbf{N}_{1}$, $\mathbf{N}_{2}, \mathbf{N}_{n}=$ número de dias de semeadura na primeira, segunda e última contagens.

Os dados foram submetidos à análise de variância. As médias da ausência e presença do craspédio e do ano de colheita foram comparadas pelo teste $F$, enquanto aquelas dos 14 tratamentos pré-germinativo, pelo teste de Scott-Knott, ambos a 5\% de probabilidade.

\section{RESULTADO E DISCUSSÃO}

As maiores porcentagens de germinação ocorreram nas sementes que foram semeadas fora dos craspédios e submetidas a tratamentos de imersão em água nas temperaturas de $70,80,90 \mathrm{e} 100^{\circ} \mathrm{C}$ por 1 min e posterior choque térmico, bem como aquelas dos tratamentos de imersão em água quente a 70,80 e $90{ }^{\circ} \mathrm{C}$ até o resfriamento total (Tabela 1).

Os tratamentos de imersão em água nas temperaturas de 90 e $100^{\circ} \mathrm{C}$ por $1 \mathrm{~min}$, com posterior choque térmico, das sementes sem craspédio destacaram-se, pois proporcionaram resultados semelhantes nos dois anos de colheita (2002 e 2003). Tais tratamentos diferiram do choque térmico a 70 e $80^{\circ} \mathrm{C}$, como também daqueles de imersão em água a 70,80 e $90^{\circ} \mathrm{C}$ até o resfriamento total (Tabela 1).

Um dos fatores limitantes à propagação da Mimosa caesalpiniifolia é a dormência profunda das sementes, o que resulta em germinação lenta e desuniforme. A impermeabilidade do tegumento à água é o fenômeno considerado por Popinigis (1985), como uma das causas mais comuns de dormência em leguminosas. Isso pode ser comprovado pela menor porcentagem de germinação obtida pelas sementes intactas (testemunha), em comparação com aquelas desprovidas do segmento do fruto.

As baixas porcentagens de emergência de plântulas obtidas com sementes imersas em água fria pelos períodos de $12,24,36,48$ e $60 \mathrm{~h}$, principalmente naquelas contidas nos craspédios, no ano de 2003 (Tabela 1), indicam provável ocorrência de algum tipo de dano de natureza patológica, uma vez que foi observado processo avançado de deterioração apenas nas sementes dos referidos tratamentos, o que lhes causou a morte.

Tabela 1 - Valores médios referentes à porcentagem de emergência de plântulas de Mimosa caesalpiniifolia Benth., oriundas de sementes dentro ou fora dos craspédios e submetidas a diferentes tratamentos, nos anos de 2002 e 2003

Table 1 - Mean values related to seedling emergence percentage of Mimosa caesalpiniifolia Benth., originated from seeds inside or outside the craspedia and submitted to different treatments, in the years 2002 and 2003

\begin{tabular}{|c|c|c|c|c|}
\hline \multirow[t]{3}{*}{ Tratamentos } & \multicolumn{4}{|c|}{ Segmento do Fruto (Craspédio) } \\
\hline & \multicolumn{2}{|c|}{ sem } & \multicolumn{2}{|c|}{ com } \\
\hline & 2002 & 2003 & 2002 & 2003 \\
\hline Im. água fria/ $12 \mathrm{~h}$ & $55,00 \mathrm{Aa} \beta$ & $35,00 \mathrm{Ba} \theta$ & $45,00 \mathrm{Ab} \beta$ & $21,50 \mathrm{Bb} \beta$ \\
\hline Im. água fria/24h & $53,50 \mathrm{~A} \beta$ & $42,00 \mathrm{Ba} \theta$ & $46,00 \mathrm{Aa} \beta$ & $23,50 \mathrm{Bb} \beta$ \\
\hline Im. água fria/36h & $48,00 \mathrm{Aa} \lambda$ & $42,00 \mathrm{Aa} \theta$ & $37,00 \mathrm{Ab} \lambda$ & $19,00 \mathrm{Bb} \beta$ \\
\hline Im. água fria/48h & $44,00 \mathrm{Aa} \lambda$ & $37,50 \mathrm{Aa} \theta$ & $43,50 \mathrm{Aa} \beta$ & $20,00 \mathrm{Bb} \beta$ \\
\hline Im. água fria/ $60 \mathrm{~h}$ & $48,50 \mathrm{Aa} \lambda$ & $41,50 \mathrm{Ba} \theta$ & $35,00 \mathrm{Ab} \lambda$ & $19,50 \mathrm{Bb} \beta$ \\
\hline Choque térmico $70^{\circ} \mathrm{C}$ & $73,50 \mathrm{Aa} \alpha$ & $52,00 \mathrm{Ba} \lambda$ & $53,00 \mathrm{Ab} \alpha$ & $34,00 \mathrm{Bb} \alpha$ \\
\hline Choque térmico $80{ }^{\circ} \mathrm{C}$ & $84,00 \mathrm{Aa} \alpha$ & $73,50 \mathrm{Ba} \beta$ & $34,50 \mathrm{Ab} \lambda$ & $35,50 \mathrm{Ab} \alpha$ \\
\hline Choque térmico $90{ }^{\circ} \mathrm{C}$ & $85,00 \mathrm{Aa} \alpha$ & $83,00 \mathrm{Aa} \alpha$ & $36,50 \mathrm{Ab} \lambda$ & $33,00 \mathrm{Ab} \alpha$ \\
\hline Choque térmico $100{ }^{\circ} \mathrm{C}$ & $83,00 \mathrm{Aa} \alpha$ & $80,50 \mathrm{Aa} \alpha$ & $13,00 \mathrm{Bb} \theta$ & $40,00 \mathrm{Ab} \alpha$ \\
\hline Resfri. total $70^{\circ} \mathrm{C}$ & $81,00 \mathrm{Aa} \alpha$ & $56,50 \mathrm{Ba} \lambda$ & $54,00 \mathrm{Ab} \alpha$ & $37,50 \mathrm{Bb} \alpha$ \\
\hline Resfri. total $80^{\circ} \mathrm{C}$ & $86,00 \mathrm{Aa} \alpha$ & $70,00 \mathrm{Ba} \beta$ & $53,00 \mathrm{Ab} \alpha$ & $42,00 \mathrm{Bb} \alpha$ \\
\hline Resfri. total $90^{\circ} \mathrm{C}$ & $85,00 \mathrm{Aa} \alpha$ & $72,50 \mathrm{Ba} \beta$ & $12,00 \mathrm{Bb} \theta$ & $41,50 \mathrm{Ab} \alpha$ \\
\hline Resfri. total $100{ }^{\circ} \mathrm{C}$ & $59,00 \mathrm{Aa} \beta$ & $51,50 \mathrm{Ba} \lambda$ & $3,00 \mathrm{Bb} \mu$ & $29,50 \mathrm{Ab} \beta$ \\
\hline Testemunha & $51,00 \mathrm{Ab} \lambda$ & $41,50 \mathrm{Ba} \theta$ & $61,50 \mathrm{Aa} \alpha$ & $27,50 \mathrm{Bb} \beta$ \\
\hline
\end{tabular}

Médias na mesma linha, seguidas das mesmas letras maiúsculas para anos em um tipo de artículo e minúsculas para variação de artículo em um dos anos, são iguais entre si, pelo teste $\mathrm{F}$ a $5 \%$ de probabilidade. As médias na mesma coluna, seguidas de mesmas letras gregas, são iguais entre si, pelo teste Scott-Knott a $5 \%$ de probabilidade. 
De forma semelhante, os tratamentos de imersão em água a $100^{\circ} \mathrm{C}$ por 1 min e posterior choque térmico e nas temperaturas de 90 e $100{ }^{\circ} \mathrm{C}$ até o resfriamento total, no ano de 2002, também apresentaram baixas porcentagens de emergência de plântulas (Tabela 1). Isso indica algum tipo de enfraquecimento das sementes, o que, provavelmente, causou a morte destas ou dificuldades para se livrarem de seus craspédios. Tal hipótese se deve ao fato de que as sementes retiradas dos craspédios e submetidas aos mesmos tratamentos conseguiram emergir.

Quanto à primeira contagem, verificou-se que as sementes contidas nos segmentos do fruto (craspédios) e, principalmente, submetidas aos tratamentos por imersão em água quente $\left(100,90\right.$ e $\left.100{ }^{\circ} \mathrm{C}\right)$ por choque térmico e até o resfriamento total, respectivamente, no ano de 2002, proporcionaram as menores porcentagens de emergência, os quais foram seguidos de imersão em água fria por 12, 24, 36, 48 e 60 h, no ano de 2003 (Tabela 2).
Os tratamentos de imersão em água fria por 12 , $24,36,48$ e 60 h, nas sementes contidas nos craspédios e colhidas em 2002 e 2003, acompanhados dos tratamentos em água quente $\left(90\right.$ e $100{ }^{\circ} \mathrm{C}$ e 80,90 e $\left.100^{\circ} \mathrm{C}\right)$ até o resfriamento total e choque térmico, respectivamente, no ano de 2002, mostraram porcentagens de emergência de plântulas inferiores aquelas da testemunha (Tabela 2) em razão possivelmente, de algum dano ao embrião da semente decorrente dos efeitos relativos aos tratamentos já citados.

Resultados positivos com imersão de sementes em água quente foram obtidos por Passos et al. (1988) com Leucaena leucocephala $\left(100{ }^{\circ} \mathrm{C}\right.$ por $\left.4 \mathrm{seg}\right)$, Varela et al. (1991) com Stryphnodendron pulcherrimum $(90$ ${ }^{\circ} \mathrm{C}$, por 5, 10 e $15 \mathrm{~min}$ ), Torres e Santos (1994) com Acacia senegal e Parkinsonia aculleata (entre 80$90^{\circ} \mathrm{C}$ ), Ribas et al. (1996) com Mimosa bimucronata $\left(80^{\circ} \mathrm{C}\right.$, seguido por esfriamento na mesma água por 24 h), Martins-Corder et al. (1999) e Smiderle et al. (2005) com Acacia mearnsii De Willd. e Teles et al. (2000) com Leucaena leucocephala (Lam.) de Wit.

Tabela 2 - Valores médios referentes à primeira contagem de emergência de plântulas de Mimosa caesalpiniifolia Benth., oriundas de sementes dentro ou fora dos craspédios e submetidas a diferentes tratamentos, nos anos de 2002 e 2003

Table 2 - Mean values related to the first count of seedling emergence of Mimosa caesalpiniifolia Benth., originated from seeds inside or outside the craspedia and submitted to different treatments, in 2002 and 2003

\begin{tabular}{|c|c|c|c|c|}
\hline \multirow[t]{3}{*}{ Tratamentos } & \multicolumn{4}{|c|}{ Segmento do Fruto (Craspédio) } \\
\hline & \multicolumn{2}{|c|}{ sem } & \multicolumn{2}{|c|}{ com } \\
\hline & 2002 & 2003 & 2002 & 2003 \\
\hline Im. água fria/12h & $50,00 \mathrm{Aa} \lambda$ & $33,00 \mathrm{Ba} \theta$ & $28,00 \mathrm{Ab} \beta$ & $11,50 \mathrm{Bb} \lambda$ \\
\hline Im. água fria/24h & $44,50 \mathrm{Aa} \lambda$ & $35,00 \mathrm{Ba} \theta$ & $23,50 \mathrm{Ab} \beta$ & $13,00 \mathrm{Bb} \lambda$ \\
\hline Im. água fria/36h & $44,00 \mathrm{Aa} \lambda$ & $38,50 \mathrm{Aa} \theta$ & $22,50 \mathrm{Ab} \beta$ & $8,50 \mathrm{Bb} \lambda$ \\
\hline Im. água fria/48h & $40,50 \mathrm{Aa} \lambda$ & $32,50 \mathrm{Ba} \theta$ & $21,50 \mathrm{Ab} \beta$ & $11,00 \mathrm{Bb} \lambda$ \\
\hline Im. água fria/60h & $44,50 \mathrm{Aa} \lambda$ & $36,50 \mathrm{Ba} \theta$ & $24,00 \mathrm{Ab} \beta$ & $10,50 \mathrm{Bb} \lambda$ \\
\hline Choque térmico $70^{\circ} \mathrm{C}$ & $71,50 \mathrm{Aa} \beta$ & $49,50 \mathrm{Ba} \lambda$ & $40,00 \mathrm{Ab} \beta$ & $21,00 \mathrm{Bb} \beta$ \\
\hline Choque térmico $80^{\circ} \mathrm{C}$ & $83,50 \mathrm{Aa} \alpha$ & $70,50 \mathrm{Ba} \beta$ & $22,50 \mathrm{Ab} \beta$ & $29,00 \mathrm{Ab} \alpha$ \\
\hline Choque térmico $90^{\circ} \mathrm{C}$ & $84,50 \mathrm{Aa} \alpha$ & $85,50 \mathrm{Aa} \alpha$ & $27,00 \mathrm{Ab} \beta$ & $29,00 \mathrm{Ab} \alpha$ \\
\hline Choque térmico $100^{\circ} \mathrm{C}$ & $86,00 \mathrm{Aa} \alpha$ & $80,00 \mathrm{Aa} \alpha$ & $12,00 \mathrm{Bb} \lambda$ & $33,00 \mathrm{Ab} \alpha$ \\
\hline Resfri. total $70{ }^{\circ} \mathrm{C}$ & $66,50 \mathrm{Aa} \beta$ & $55,00 \mathrm{Ba} \lambda$ & $47,50 \mathrm{Ab} \alpha$ & $30,00 \mathrm{Bb} \alpha$ \\
\hline Resfri. total $80^{\circ} \mathrm{C}$ & $86,00 \mathrm{Aa} \alpha$ & $68,00 \mathrm{Ba} \beta$ & $44,00 \mathrm{Ab} \alpha$ & $34,00 \mathrm{Bb} \alpha$ \\
\hline Resfri. total $90{ }^{\circ} \mathrm{C}$ & $84,00 \mathrm{Aa} \alpha$ & $72,50 \mathrm{Ba} \beta$ & $11,00 \mathrm{Bb} \lambda$ & $37,00 \mathrm{Ab} \alpha$ \\
\hline Resfri. total $100{ }^{\circ} \mathrm{C}$ & $56,00 \mathrm{Aa} \lambda$ & $51,50 \mathrm{Aa} \lambda$ & $3,00 \mathrm{Bb} \lambda$ & $22,00 \mathrm{Ab} \beta$ \\
\hline Testemunha & $48,00 \mathrm{Aa} \lambda$ & $37,50 \mathrm{Ba} \theta$ & $38,00 \mathrm{Ab} \alpha$ & $18,50 \mathrm{Bb} \beta$ \\
\hline
\end{tabular}

Médias na mesma linha, seguidas das mesmas letras maiúsculas para anos em um tipo de artículo e minúsculas para variação de artículo em um dos anos, são iguais entre si, pelo teste $\mathrm{F}$ a $5 \%$ de probabilidade. As médias na mesma coluna, seguidas de mesmas letras gregas, são iguais entre si, pelo teste Scott-Knott a 5\% de probabilidade. 
Embora seja um método vantajoso para superar a dormência em sementes de leguminosas pelo baixo custo, a água fervente tem proporcionado resultados contraditórios (RODRIGUES et al., 1990). Para Stryphnodendron pulcherrimum (VARELA et al., 1991) e Mimosa caesalpiniaefolia (MARTINS et al., 1992), a água fervente foi letal. Com Senna macranthera (colladon) Irwin \& Barneby, os tratamentos com água quente foram menos eficientes do que aqueles com ácido sulfúrico (SANTARÉM e ÁQUILA, 1995). Os tratamentos de imersão em água quente por 5, 10 e 15 min inibiram a germinação das sementes de Copaifera Langsdorffii (PEREZ e PRADO, 1993) e não foram eficientes para superar a dormência das sementes de espécies de Cassia estudadas (RODRIGUES et al., 1990). De forma semelhante, Grus et al. (1984) também constataram que o tratamento de imersão em água fervente por $1,2,3$ e $5 \mathrm{~min}$ foram prejudiciais a Caesalpinia leiostachya (Benth.), Ducke e Cassia javanica Ried, matando ou danificando o embrião.

Os maiores índices de velocidade de emergência foram obtidos com as sementes semeadas fora do craspédio e imersas em água quente $\left(80,90\right.$ e $\left.100{ }^{\circ} \mathrm{C}\right)$ por $1 \mathrm{~min}$, com posterior choque térmico e com aquelas submetidas aos tratamentos de imersão em água nas temperaturas de 80 e $90{ }^{\circ} \mathrm{C}$ até o resfriamento total, nas sementes de 2002. No entanto, destacaram-se os tratamentos de imersão em água quente $\left(90^{\circ} \mathrm{C}\right.$ e 100 $\left.{ }^{\circ} \mathrm{C}\right)$ com posterior choque térmico, por terem proporcionado altos índices de velocidade de emergência, tendo estes se apresentado semelhantes nos dois anos (2002 e 2003). Já nas sementes contidas nos segmentos dos craspédios, todos os tratamentos indicaram resultados inferiores (Tabela 3).

Tabela 3 - Valores médios referentes ao índice de velocidade de emergência de plântulas (IVE) de Mimosa caesalpiniifolia Benth., oriundas de sementes dentro ou fora dos craspédios e submetidas a diferentes tratamentos, nos anos de 2002 e 2003

Table 3 - Mean values of seedling emergence speed index (IVE) of Mimosa caesalpiniifolia Benth., originated from seeds inside or outside the craspedia and submitted to different treatments in 2002 and 2003

\begin{tabular}{|c|c|c|c|c|}
\hline \multirow[t]{3}{*}{ Tratamentos } & \multicolumn{4}{|c|}{ Segmento do Fruto (Craspédio) } \\
\hline & \multicolumn{2}{|c|}{ sem } & \multicolumn{2}{|c|}{ com } \\
\hline & 2002 & 2003 & 2002 & 2003 \\
\hline Im. água fria/ $12 \mathrm{~h}$ & $5,63 \mathrm{Aa} \lambda$ & $3,21 \mathrm{Ba} \lambda$ & $2,79 \mathrm{Ab} \beta$ & $1,11 \mathrm{Bb} \lambda$ \\
\hline Im. água fria/24h & $5,13 \mathrm{Aa} \theta$ & $3,93 \mathrm{Ba} \lambda$ & $2,69 \mathrm{Ab} \beta$ & $1,38 \mathrm{Bb} \lambda$ \\
\hline Im. água fria/36h & $5,49 \mathrm{Aa} \lambda$ & $4,01 \mathrm{Ba} \lambda$ & $2,35 \mathrm{Ab} \beta$ & $1,01 \mathrm{Bb} \lambda$ \\
\hline Im. água fria/48h & $4,18 \mathrm{Aa} \theta$ & $3,45 \mathrm{Aa} \lambda$ & $2,80 \mathrm{Ab} \beta$ & $1,26 \mathrm{Bb} \lambda$ \\
\hline Im. água fria/ $60 \mathrm{~h}$ & $4,85 \mathrm{Aa} \theta$ & $4,03 \mathrm{Aa} \lambda$ & $2,22 \mathrm{Ab} \beta$ & $1,18 \mathrm{Bb} \lambda$ \\
\hline Choque térmico $70^{\circ} \mathrm{C}$ & $8,38 \mathrm{Aa} \beta$ & $5,68 \mathrm{Ba} \beta$ & $3,30 \mathrm{Ab} \alpha$ & $2,14 \mathrm{Bb} \lambda$ \\
\hline Choque térmico $80^{\circ} \mathrm{C}$ & $10,29 \mathrm{Aa} \alpha$ & $8,87 \mathrm{Ba} \alpha$ & $2,12 \mathrm{Ab} \beta$ & $2,61 \mathrm{Ab} \alpha$ \\
\hline Choque térmico $90{ }^{\circ} \mathrm{C}$ & $9,73 \mathrm{Aa} \alpha$ & $9,84 \mathrm{Aa} \alpha$ & $2,43 \mathrm{Ab} \beta$ & $2,60 \mathrm{Ab} \alpha$ \\
\hline Choque térmico $100{ }^{\circ} \mathrm{C}$ & $10,34 \mathrm{Aa} \alpha$ & $9,89 \mathrm{Aa} \alpha$ & $0,95 \mathrm{Bb} \lambda$ & $2,83 \mathrm{Ab} \alpha$ \\
\hline Resfri. total $70^{\circ} \mathrm{C}$ & $8,99 \mathrm{Aa} \beta$ & $6,21 \mathrm{Ba} \beta$ & $4,21 \mathrm{Ab} \alpha$ & $2,95 \mathrm{Bb} \alpha$ \\
\hline Resfri. total $80{ }^{\circ} \mathrm{C}$ & $10,68 \mathrm{Aa} \alpha$ & $6,71 \mathrm{Ba} \beta$ & $3,77 \mathrm{Ab} \alpha$ & $2,97 \mathrm{Ab} \alpha$ \\
\hline Resfri. total $90{ }^{\circ} \mathrm{C}$ & $10,63 \mathrm{Aa} \alpha$ & $9,52 \mathrm{Ba} \alpha$ & $0,93 \mathrm{Bb} \lambda$ & $3,47 \mathrm{Ab} \alpha \alpha$ \\
\hline Resfri. total $100{ }^{\circ} \mathrm{C}$ & $6,74 \mathrm{Aa} \lambda$ & $6,85 \mathrm{Aa} \beta$ & $0,27 \mathrm{Bb} \lambda$ & $2,06 \mathrm{Ab} \lambda$ \\
\hline Testemunha & $4,88 \mathrm{Aa} \theta$ & $3,89 \mathrm{Ba} \lambda$ & $3,45 \mathrm{Ab} \alpha$ & $1,82 \mathrm{Bb} \lambda$ \\
\hline
\end{tabular}

Médias na mesma linha, seguidas das mesmas letras maiúsculas para anos em um tipo de artículo e minúsculas para variação de artículo em um dos anos, são iguais entre si, pelo teste $\mathrm{F}$ a $5 \%$ de probabilidade. As médias na mesma coluna, seguidas de mesmas letras gregas, são iguais entre si, pelo teste Scott-Knott a $5 \%$ de probabilidade.

\section{CONCLUSÕES}

O tratamento de imersão em água quente (90 $\left.{ }^{\circ} \mathrm{C}\right)$ com posterior choque térmico, nas sementes mais velhas (2002), seguido do mesmo tratamento a 100 ${ }^{\circ} \mathrm{C}$, em sementes que foram semeadas fora dos craspédios, mostrou-se mais adequados para a condução dos testes de germinação e vigor em sementes de Mimosa caesalpiniifolia Benth.

As sementes sem craspédios tiveram melhor qualidade fisiológica.

A homogeneidade do processo germinativo é maior nas sementes que foram semeadas fora dos craspédios, independentemente do ano empregado. 


\section{REFERÊNCIAS}

AZERÊDO, G. A. et al. Desempenho de sementes de sapoti (Achras sapota L.) submetidas a diferentes tratamentos pré-germinativos. Revista Brasileira de Fruticultura, v.24, n.1, p.147-150, 2002.

BARBOSA, A. P. et al. Tecnologia alternativa para a quebra de dormência das sementes de pau-debalsa (Ochroma lagopus Sw., Bombacaceae).

Acta Amazonica, v.34, n.1, p.107-110, 2004.

BRASIL. Ministério da Agricultura e Reforma Agrária. Regras para análise de sementes. Brasília: SNDA/DNDV/CLAV, 1992.365p.

\section{CANDIDO, J. F. et al. Orelha-de-negro}

(Enterolobium contortisiliquum (Vell.) Morong.): dormência e métodos para sua quebra. Revista Árvore, v.8, n.2, p.104-10, 1982.

CARVAlho, N. M.; NAKAGAWA, J. Sementes: ciência, tecnologia e produção. 4.ed. Jaboticabal: FUNEP, 2000. 588p.

CÍCERO, S. M. Dormência de sementes. In: SEMANA DEATUALIZAÇÃOEMPRODUÇÃODESEMENTES, 1., 1986, Campinas. Trabalhos...Campinas: Fundação Cargill, 1986.p.41-76.

Costa, M. G. O Sabiá. Areia: Centro de Ciências Agrárias da Universidade Federal da Paraíba - CCA/UFPB, 1983. (Boletim Técnico, 4)

EIRA, M. T. S.; FREITAS, R. W. A.; MELLO, C. M..C. Superação da dormência de sementes de Enterolobium contortisiliquum (Vell.) Morong. Leguminosae. Revista Brasileira de Sementes, v.15, n.2, p.177-181, 1993.

GRUS, V. M.; DE MATTE, M. E. S. P.; GRAZIANO, T. T. Germinação de sementes de pau-ferro e cássiajavanesa submetidas a tratamentos para quebra de dormência. Revista Brasileira de Sementes, v.6, n.2, p.29-35, 1984.

HARTMANN, H. T.; KESTER, D. E., Propagación de planta: principios y prácticas. México: Continental, 1978.810p.
KRAMER, P. J.; KOZLOZWISKI, T. T.

Fisiologia das árvores. Lisboa: Fundação Calouste Gulbenkian, 1972. 745p.

LEMOS FILHO, J. P. et al. Germinação de sementes de Senna macranthera, Senna multijuga e Stryphnodendron polyphyllum. Pesquisa Agropecuária Brasileira, v.32, n.4, p.357-361, 1997.

LIMA, D.; GARCIA, L. C. Avaliação de métodos para teste de germinação de sementes de Acacia mangium Willd. Revista Brasileira de Sementes, v.18, n.2, p.180-185, 1996.

MAGUIRE, J. D. Speed of germination aid in selection and evaluation for seedling emergence and vigor. Crop Science, v.2, n.2, p.176-177, 1962.

MARTINS, C. C.; CARVALHO, N. M.; OLIVEIRA, A. P. Quebra de dormência de sementes de sabiá (Mimosa caesalpiniaefolia Benth.). Revista Brasileira de Sementes, v.14, n.1, p.5-8, 1992.

MARTINS-CORDER, M. P.; BORGES JUNIOR, N. Desinfestação e quebra de dormência de sementes de Acacia mearnsii de Wild. Ciência Florestal, v.9, n.2, p.1-7, 1999.

MARTINS-CORDER, M. P.; BORGES, R. Z.; BORGES JUNIOR, N. Fotoperiodismo e quebra de dormência em sementes de acácia negra (Acacia mearnsii De Wild.). Ciência Florestal, v.9, n.1, p.71-77, 1999.

OLIVEIRA, L. M.; DAVIDE,A. C.; CARVALHO, M.L. M. Avaliação de métodos para quebra da dormência e para a desinfestação de sementes de canafístula (Peltophorum dubium (Sprengel) Taubert. Revista Árvore, v.27, n.5, p.597-603, 2003.

PASSOS, M. A. A.; LIMA, T. V.; ALBUQUERQUE, J. L. Quebra de dormência de sementes de leucena. Revista Brasileira de Sementes, v.10, n.2, p.97-102, 1988.

PEREZ, S. C. J. G. A.; PRADO, C. H. B. A. Efeitos de diferentes tratamentos pré-germinativos e da concentração de alumínio no processo germinativo de sementes de Copaifera langsdorfii Desf. Revista Brasileira de Sementes, v.15, n.1, p.115-118, 1993.

R. Árvore, Viçosa-MG, v.32, n.2, p.203-210, 2008 
POPINIGIS, F. Fisiologia da semente. 2.ed. Brasília: ABRATES, 1985. 298p.

RIBAS, L. L. F.; FOSSATI, L. C.; NOGUEIRA, A. C. Superação da dormência de sementes de Mimosa bimucronata (D.C.) O.Kuntze (maricá).

Revista Brasileira de Sementes, v.18, n.1, p.98-101, 1996.

RIBEIRO, D. V. Programa de produção e tecnologia de sementes de espécies florestais nativas e exóticas desenvolvido pela estação florestal de experimentação Eng. Ag. Mário Xavier. In: SIMPÓSIO BRASILEIRO SOBRE TECNOLOGIA DE SEMENTES FLORESTAIS, 1., 1984, Belo Horizonte. Anais... Brasília: IBDF, 1984. p.109-118.

RIZZINI, C. T. Árvores e madeiras úteis do Brasil: manual de dendrologia brasileira. São Paulo: Edgard Blucher, 1971. 294p.

RODRIGUES, E. H. A.; AGUIAR, I. B.; SADER, R. Quebra de dormência de sementes de três espécies do gênero Cassia. Revista Brasileira de Sementes, v.12, n.12, p.17-25, 1990.

SANTARÉM, E. R.; ÁQUILA, M. E. A. Influência de métodos de superação de dormência e do armazenamento na germinação de sementes de Senna mancranthera (Colladon) Irwin \& Barneby (Leguminosae). Revista Brasileira de Sementes, v.17, n.2, p.205-209, 1995.
SCHEFFER-BASSO, S. M.; VENDRUSCULO, M. C. Germinação de sementes das leguminosas forrageiras nativas Adesmia araujoi Burk. E Desmodium incanum D.C. Revista Brasileira de Agrociência, v.3, n.2, p.65-68, 1997.

SMIDERLE, O. J.; MOURÃO JUNIOR, M.; SOUSA, R. C. P. Tratamentos pré-germinativos em sementes de acácia. Revista Brasileira de Sementes, v.27, n.1, p.78-85, 2005.

TELES, M. M. et al.. Métodos para quebra da dormência em sementes de leucena (Leucaena leucocephala (Lam.) de Wit. Revista Brasileira de Zootecnia, v.29, n.2, p.387-391, 2000.

TORRES, S. B.; SANTOS, S. S. B. Superação da dormência em sementes de Acacia senegal (L.) Willd. e Parkinsonia aculeata L. Revista Brasileira de Sementes, v.16, n.1, p.54-57, 1994.

VARELA, V. P.; BROCKI, E.; SÁ, S. T. V. Tratamentos pré-germinativos de espécies da amazônia. IV. Faveira camuzê - Stryphnodendron pulcherrimum (Willd.) Hochr. - Leguminosae. Revista Brasileira de Sementes, v.13, n.2, p.87-89, 1991.

VAZQUEZ-YANES, C.; OROZCO-SEGOVIA,A. Patterns of seed longevity and germination in the tropical rainforest. Annual Review of ecology and Systematics, v.24, n.1, p.69-87, 1993.

WILlan, R. L. Seed pretreatment.

Humleaback: Danida Forest Seed Centre, 1990. 19p. (Lecture Note). 